\title{
Lesioni, malattia, paziente
}

Giovanni Lodi

Dipartimento di Scienze Biomediche, Chirurgiche e Odontoiatriche

Università degli Studi di Milano

via Beldiletto 1/3 Milano 20142

Italia

giovanni.lodi@unimi.it

tel. + 390250319021

fax. +390250319041

La medicina e l'odontoiatria devono essere al servizio del paziente o, se preferite, centrate sul paziente. Nessun dubbio a riguardo.

Sfortunatamente questa espressione viene spesso usata a sproposito, talvolta con un significato un po' vago e, se associata a parole eccellenti come "olistica" o "naturale", risulta un po' troppo new age per i miei gusti. Che però, in quanto tali, sono opinabili.

Ho partecipato qualche settimana fa a un piccolo grande congresso, organizzato da un collega tanto bravo quanto appassionato. Congresso che si è svolto in un clima mite, non nel senso metereologico (pioveva), ma perché neolaureati ed esperti, odontoiatri e igienisti, specialisti e generalisti hanno confrontato opinioni, dati della ricerca e pratiche cliniche in maniera pacata, gentile e alla pari.

Qui ho avuto la fortuna di ascoltare due colleghi che hanno illustrato con grande chiarezza uno dei modi in cui è possibile declinare una pratica centrata sul paziente. II primo, un parodontologo, ha raccontato come la sua storia professionale lo abbia portato a concludere che l'obiettivo principale non sia curare tasche o ridurle di pochi millimetri con difficili interventi chirurgici (pratiche peraltro meritorie), tanto meno estrarre denti per sostituirli con impianti.

Perché ha realizzato quanto sia meglio curare la malattia parodontale piuttosto che i suoi esiti. II secondo, un cariologo, dopo aver messo in imbarazzo la platea (sapete elencare almeno tre famosi ricercatori nel campo della cariologia?) ha spiegato che è la carie (nel senso della malattia) che dobbiamo curare, perché non faremmo un gran servizio ai nostri pazienti se ci limitassimo a trattare le carie (nel senso delle lesioni cariose).

Rimarcando così anche l'ambiguità del termine carie e la confusione che può generare. Ma come si fa a curare malattia parodontale e carie? La risposta dei due colleghi è la medesima: bisogna passare dal paziente e dalla sua determinazione a tutelare la propria salute.

Perché solo corrette pratiche di igiene orale, eliminazione dei fattori di rischio modificabili, interventi sulla dieta e una vera alleanza terapeutica, possono curare queste due malattie.

La cura di queste malattie, le nostre malattie, è quindi necessariamente centrata sul paziente.

Buona lettura 